\title{
Archéopages
}

Archéopages

Archéologie et société

42 | 04-07/2015

Construire en terre crue

\section{La brique crue, matériau par défaut ou choix éclairé?}

Le cas des fours de tuilier antiques de Mours (Val-d'Oise)

The Roman tile kilns built of mud bricks from Mours (Val-d'Oise). A default

material or an enlightened choice?

Los hornos tejeros antiguos de ladrillos de barro en Mours (Valle del Oise)

¿Material por descarte o decisión intencional?

\section{Aurélia Alligri et Pascal Raymond}

\section{OpenEdition}

Journals

Édition électronique

URL : https://journals.openedition.org/archeopages/1237

DOI : 10.4000/archeopages. 1237

ISSN : 2269-9872

Éditeur

INRAP - Institut national de recherches archéologiques préventives

Édition imprimée

Date de publication : 1 mars 2016

Pagination : 78-87

ISSN : 1622-8545

Référence électronique

Aurélia Alligri et Pascal Raymond, "La brique crue, matériau par défaut ou choix éclairé ? ",

Archéopages [En ligne], 42 | 04-07/2015, mis en ligne le 01 juillet 2017, consulté le 02 juin 2021. URL: http://journals.openedition.org/archeopages/1237 ; DOI : https://doi.org/10.4000/archeopages. 1237 


\title{
La brique crue, matériau
}

\section{par défaut ou choix éclairé? Le cas des fours de tuilier antiques de Mours (Val-d'Oise)}

\author{
Aurélia Alligri ${ }^{\text {Irrap }}$ \\ Pascal Raymond Inrap
}

Le site archéologique de Mours, « le Derrière des Moulins » ${ }^{\mathbf{1}}$ (Mondoloni, 2015), est localisé en bordure de la plaine alluviale de l'Oise, à 2,7 km à l'ouest de la voie romaine Paris-Beauvais sur laquelle vient s'implanter la ville antique de Beaumont-sur-Oise. La période antique est caractérisée par deux occupations successives : l'implantation d'un atelier de tuilier dès l'époque augustéenne puis, du $\mathrm{I}^{\mathrm{er}}$ au $\mathrm{III}^{\mathrm{e}}$ siècle, l'installation d'un établissement matérialisé par des bâtiments en pierre [ill. 1]. La fouille exhaustive des deux fours de tuilier (1009 et 2572) [ill. 2] a permis de révéler une architecture complexe composée de briques crues et d'éléments en terre cuite (Alligri et Raymond, 2015). La présence de ce type de vestiges en Île-de-France est assez exceptionnelle. Le fait qu'ils soient en partie construits avec des briques crues l'est davantage encore. Ce matériau de construction, largement utilisé sur le pourtour méditerranéen, est assez insolite sur les sites antiques d'île-de-France. Les découvertes récentes montrent toutefois qu'il est maintenant identifié dans divers contextes (voir article de G. BruleyChabot dans ce volume).

Les fours découverts à Mours présentent une architecture soignée et parfaitement maîtrisée qui nous interpelle sur le choix des matériaux et leur mode de mise en œuvre. Le constructeur a-t-il employé la terre crue par défaut, en fonction de ses moyens, ou sont-ce les propriétés mêmes du matériau qui ont motivé son utilisation?

\section{Architecture des fours}

Comparables à d'autres structures recensées dans le monde romain (Charlier, 2011), les fours de Mours sont à tirage vertical, construits tous deux selon des proportions identiques, avec pour chacun un foyer enterré et une fosse de travail en pente douce jusqu'à l'entrée de l'alandier [ill. 3]. Leur singularité repose sur une utilisation de la terre crue comme matériau de construction. Un exemple comparable dans l'emploi massif de ce matériau est localisé à Neupotz en RhénaniePalatine (Allemagne) (Schulz et Fischer, 2002). Quant à l'organisation spatiale de cette unité de production de terre cuite architecturale, il faut également se rendre en Allemagne, à Rheinzabern, pour trouver un exemple proche (Trimpert, 2003). Par ailleurs, l'atelier de potiers de Bastide-Neuve à Velaux (Bouches-du-Rhône) montre un exemple similaire de par sa datation et le choix des matériaux puisqu'un des fours y est construit en brique crue (Mauné et Silvéréano, 2011).

Les chambres de chauffe de forme rectangulaire sont excavées dans un encaissant marno-calcaire. Elles étaient couvertes des laboratoires aujourd'hui disparus. L'état de conservation des maçonneries formant les élévations des alandiers et des chambres de chauffe n'est pas équivalent pour les deux structures. Le four 1009 est presque entièrement récupéré, tandis que l'architecture du four 2572 est quasi complète. Notre réflexion concernant les matériaux de construction s'est donc principalement fondée sur nos observations du four 2572. On notera tout de même que l'emploi des briques crues est attesté pour le four $1009 \mathrm{au}$ niveau de ses deux massifs d'entrée et d'une partie de son alandier encore en place.

Le four 2572, de type IIE (Le Ny, 1988), est composé de trois éléments structurants : un alandier encadré par deux imposants massifs, les murs périphériques de la chambre de chauffe et des aménagements internes pour supporter la charge à cuire [ill. 2]. L'entrée de l'alandier (N) est 
de la période médiévale,

parmi lesquels deux fours

antiques (1009 et 2572) et

des structures associées.

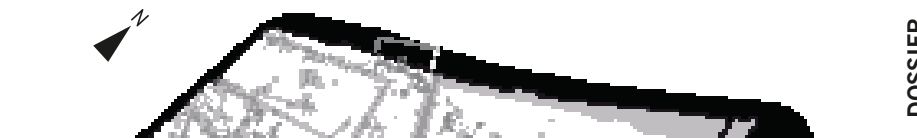

\section{$\square$ Fours}

Structures associées au fours

Autres structures

- Limite de décapage
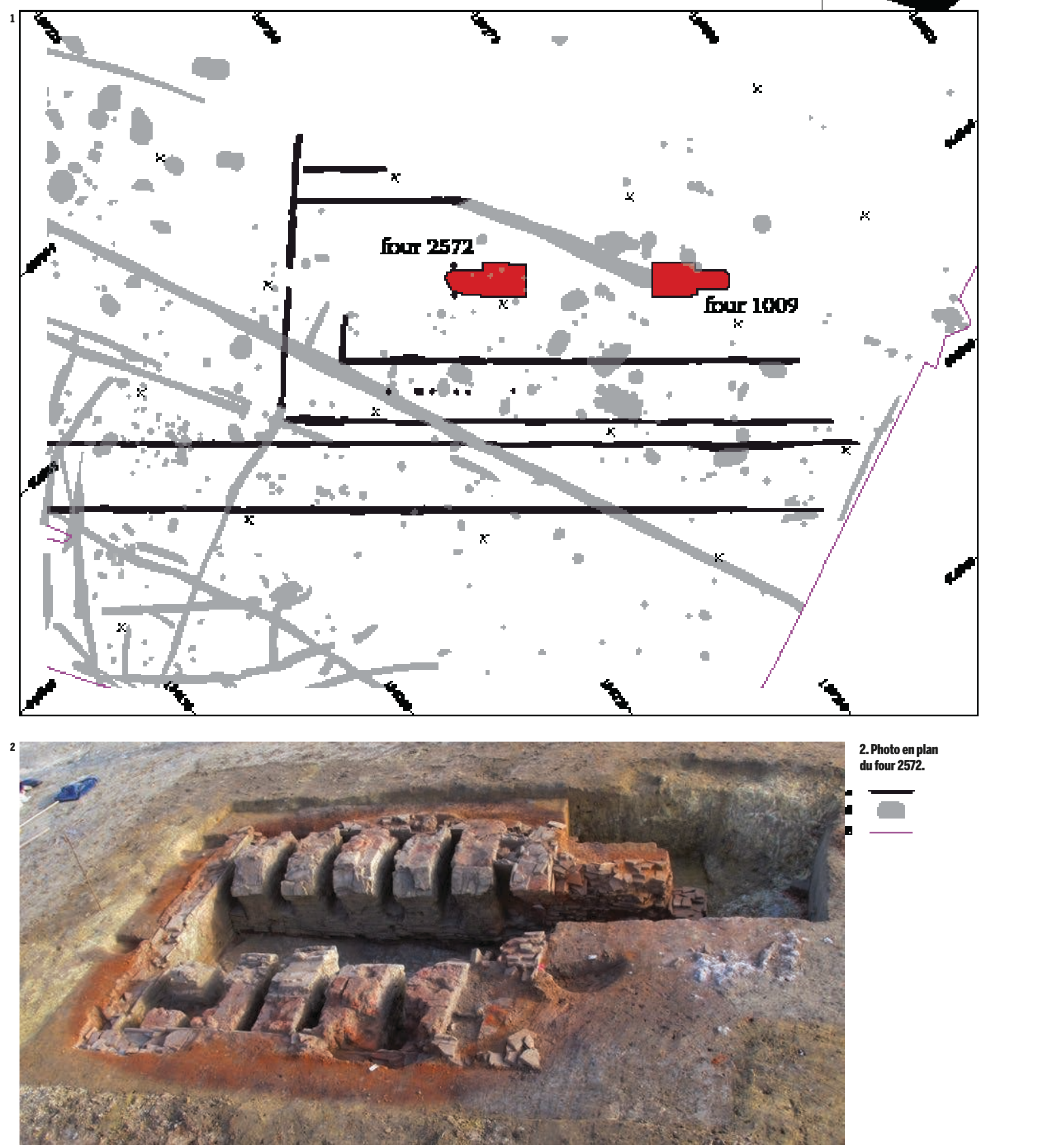

2. Photo en plan du four 2572. 

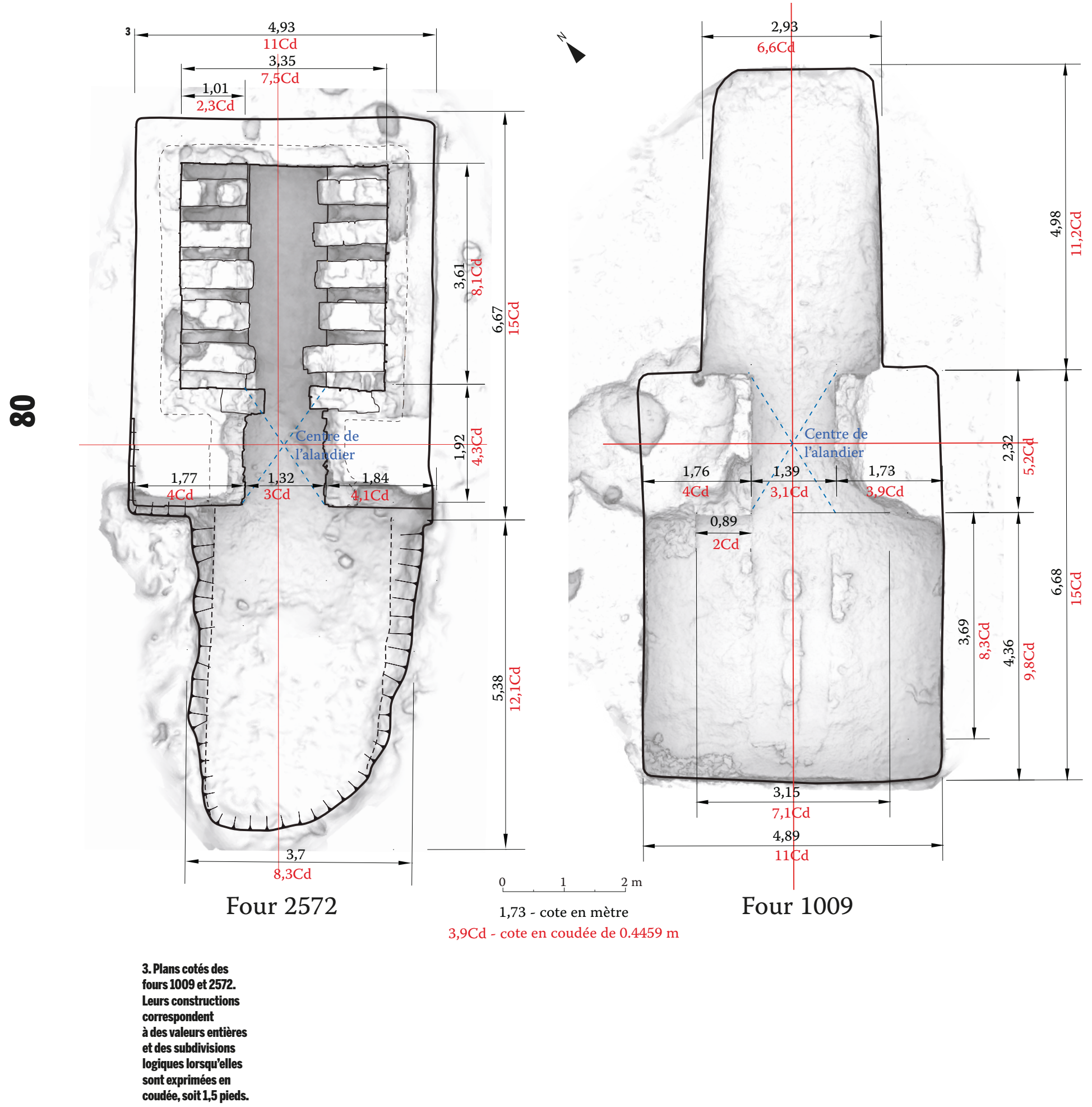
marquée par deux piédroits de tegulce empilées à plat. Il se poursuit par un couloir maçonné avec des fragments de tegulce et couvert par une voûte en berceau (J et K). Ce couloir de chauffe est encadré par deux massifs de briques crues dont le parement est en boutisse et panneresse selon un rythme irrégulier (L et M) [ill. 4a]. L'alandier et les massifs sont bâtis sur un radier de briques crues posées à plat directement sur le fond de l'excavation [ill. 4b-projections II et III]. Les murs périphériques de la chambre de chauffe (A, G, H et I), qui sont liés à l'alandier, sont construits en briques crues avec, en parement, des fragments de tegulce [ill. 4a]. Ces murs sont posés directement sur le fond du creusement sans préparation particulière [ill. 4b-projections I et II].

Les aménagements internes de la chambre de chauffe sont quant à eux construits sans chaînage avec le reste de la substructure et viennent simplement en appui sur les murs périphériques [ill. 4a]. Comme pour les massifs d'entrée, ces aménagements sont bâtis sur un radier de brique crue parallèlement au canal de chauffe [ill. 4b-projection II]. Ces élévations de faible hauteur forment deux banquettes latérales de briques crues parementées avec des tegulc. Elles supportent cinq arcs plein cintre (B à F). Cette succession dense d'arcs a pour fonction de supporter la production de terres cuites et de faciliter le flux d'air chaud.

\section{Choix des matériaux}

Cette alternance entre élément cru et cuit soulève un certain nombre d'interrogations. Les matériaux de construction connus à l'époque romaine ont des caractéristiques variées qui laissent aux bâtisseurs une grande latitude sur les alternatives dont ils disposent pour édifier leurs ouvrages. Un four de tuilier est, d'un certain point de vue, une construction comme les autres, mais sa spécificité fonctionnelle en fait une structure plus complexe à bâtir. En effet, en plus des contraintes de charges permanentes, un four doit pouvoir supporter une charge d'exploitation importante. C'est aussi une structure technique soumise à de très fortes températures. Les niveaux atteints au cours des cuissons sont tels que les transformations physicochimiques des matériaux peuvent se traduire par l'affaiblissement de leur résistance mécanique. Ainsi, des matériaux mal choisis peuvent conduire à une dégradation rapide et irrémédiable de la structure. Il est donc indispensable que le choix des matériaux soit mûrement réfléchi pour répondre aux contraintes auxquelles la structure sera soumise.

Les calcaires et grès, observés sur le site dans des constructions voisines aux deux fours, ont l'avantage d'être accessibles sans difficulté dans un environnement immédiat. Cette facilité d'approvisionnement n'a pourtant pas été un critère de choix pour les bâtisseurs puisque aucune pierre n'est employée dans les deux fours. Les raisons sont sans doute dues à la trop forte dégradation de la résistance des roches lorsqu'elles subissent des élévations de température. En effet, les variations de température correspondent toujours à un changement de volume de la matière. C'est notamment le cas des roches riches en quartz, comme les grès, où un point critique se situe à $575^{\circ} \mathrm{C}$. À cette température, le quartz change d'état et se dilate considérablement. Ainsi, la pierre léchée par les flammes se réchauffe vite en surface tandis que la diffusion lente de cette chaleur vers l'intérieur de la pierre entraîne des différences de température dans le bloc. Ces dernières provoquent alors des tensions supérieures à la résistance du matériau qui conduisent à sa fracturation. Ainsi, selon les roches, leur résistance à la compression décroît pour ne plus représenter, autour des $600{ }^{\circ} \mathrm{C}$, que $15 \%$ à $50 \%$ de ce qu'elle était à $20^{\circ} \mathrm{C}$ (HomandÉtienne, 1986). L'abaissement du point de rupture dans ces proportions devient une contrainte forte et rend les pierres inutilisables dans un milieu où les températures atteintes oscillent autour de $800^{\circ} \mathrm{C}$.

La terre cuite, quant à elle, a acquis lors de sa fabrication des capacités de résistance au feu que les autres matériaux ne possèdent pas. Son atout majeur réside dans sa capacité de résistance à la compression qui reste quasiment stable jusqu'à $750^{\circ} \mathrm{C}$. Au-delà de cette température, sa plasticité augmente et sa résistance diminue mais jamais au point d'atteindre la rupture (Nguyen, 2009). Les températures nécessaires à la cuisson des briques et des tuiles (autour des $800^{\circ} \mathrm{C}$ ) n'entraînent donc aucune modification fondamentale des éléments en terre cuite composant l'architecture des fours. À Mours, les constructeurs ont choisi de n'utiliser la terre cuite qu'aux endroits stratégiques du four. Principalement à deux emplacements : là où le flux d'air chaud est en contact avec la structure et là où les contraintes mécaniques exigent des matériaux capables de les supporter. C'est notamment le cas pour la construction des arcs supportant la charge à cuire. Les forces exercées ne sont pas seulement dirigées verticalement. Les poussées y sont redirigées latéralement et les contraintes sur les matériaux sont plus complexes. Les tegulce ou les briques cuites qui composent ces arcs sont ici les matériaux parfaitement adaptés pour encaisser ces charges [ill. 4b-projection I]. Cette capacité est liée à la modification de la structure cristalline des éléments en terre cuite. Ceux-ci augmentent ainsi leur résistance en traction à haute température en raison des caractéristiques visqueuses de leur composant silice. Dans les fours de tuilier de Mours, les températures n'ont jamais atteint les niveaux où l'état plastique des briques leur fait perdre totalement leur résistance. La terre cuite est également utilisée comme revêtement pour protéger la maçonnerie ( $\mathrm{G}, \mathrm{H}$, et I). Comme pour l'alandier, des fragments de tegula sont posés à plat et offrent en parement leur rebord latéral [ill. 4b-projection II et ill. 5b]. Ce montage sans joint constitue une surface lisse et résistante qui est exposée aux feux. Il ne s'agit pas là d'un contremur, mais d'une chemise qui semble montée 
4a. Plan du four 2572;

b. Projections du four

2572.

๙ projection

projection IV

projection

projection III-

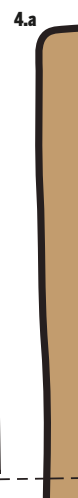

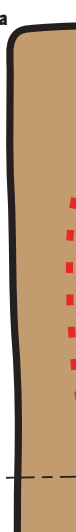

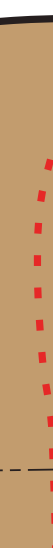

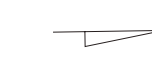

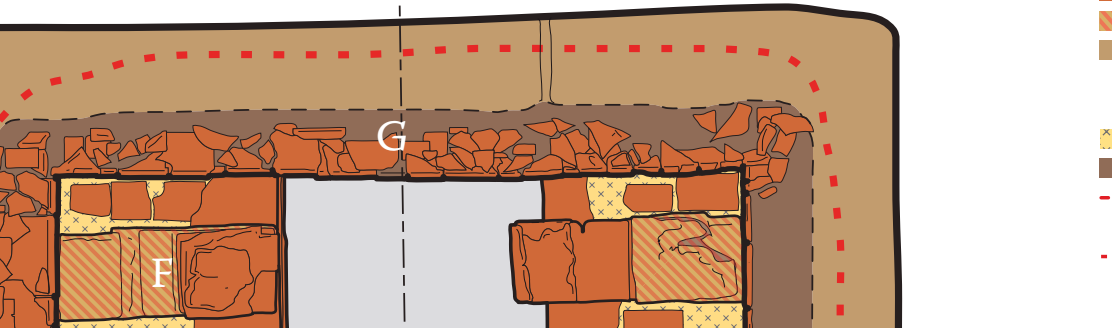

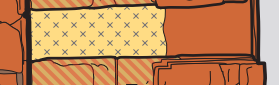

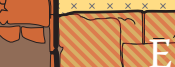

TU NE

$\mathrm{H}$

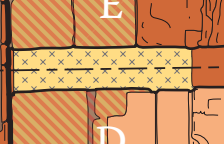

ID

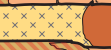

C.

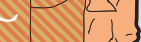

$\left.x^{x} x^{x} x^{x} x^{x}\right\}$

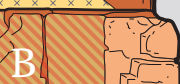

Brique crue rubéfiée

Brique crue

Maçonnerie en brique

crue peu lisible en plan

$\times \times$ Enduit d'argile

Mortier de terre

- Limite d'induration

du canal de chauffe

- Limite de rubéfaction

Rampe d'accès

N Canal de chauffe

J-K Parement de l'alandier

L-M Massif en brique crue

A-G-H-I Chemise de la chambre de chauffe en tegulae

$\mathbf{B}$ à $\mathbf{F}$ Cloison support Du laboratoire 


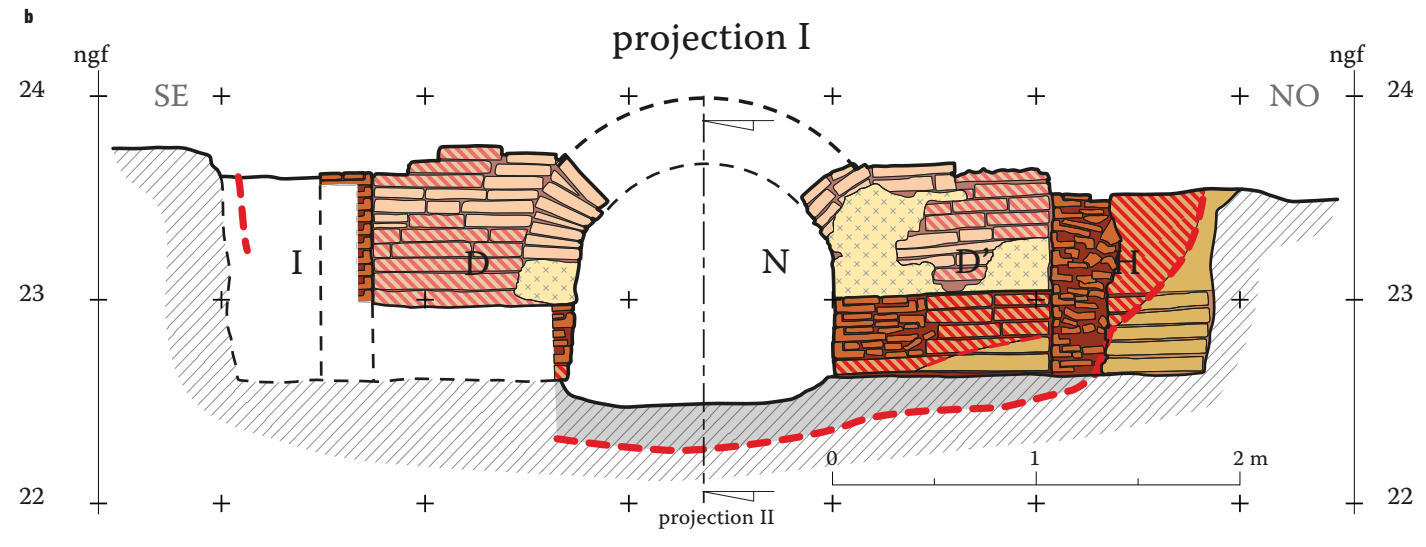

Matériaux en parement

Brique

Nivique crue rubéfiée

x.x.x Enduit d'argile

Matériaux en coupe

Brique

Tegulce

Brique crue

IN Brique crue rubéfiée

--- Limite de rubéfaction

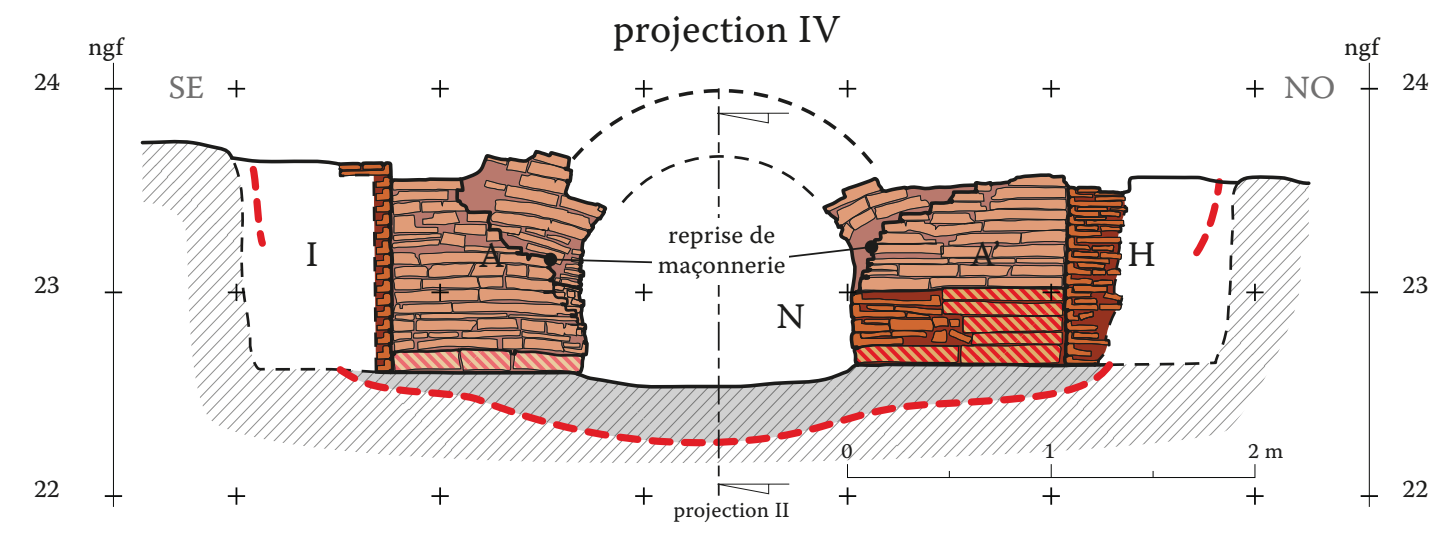

$\dddot{\infty}$
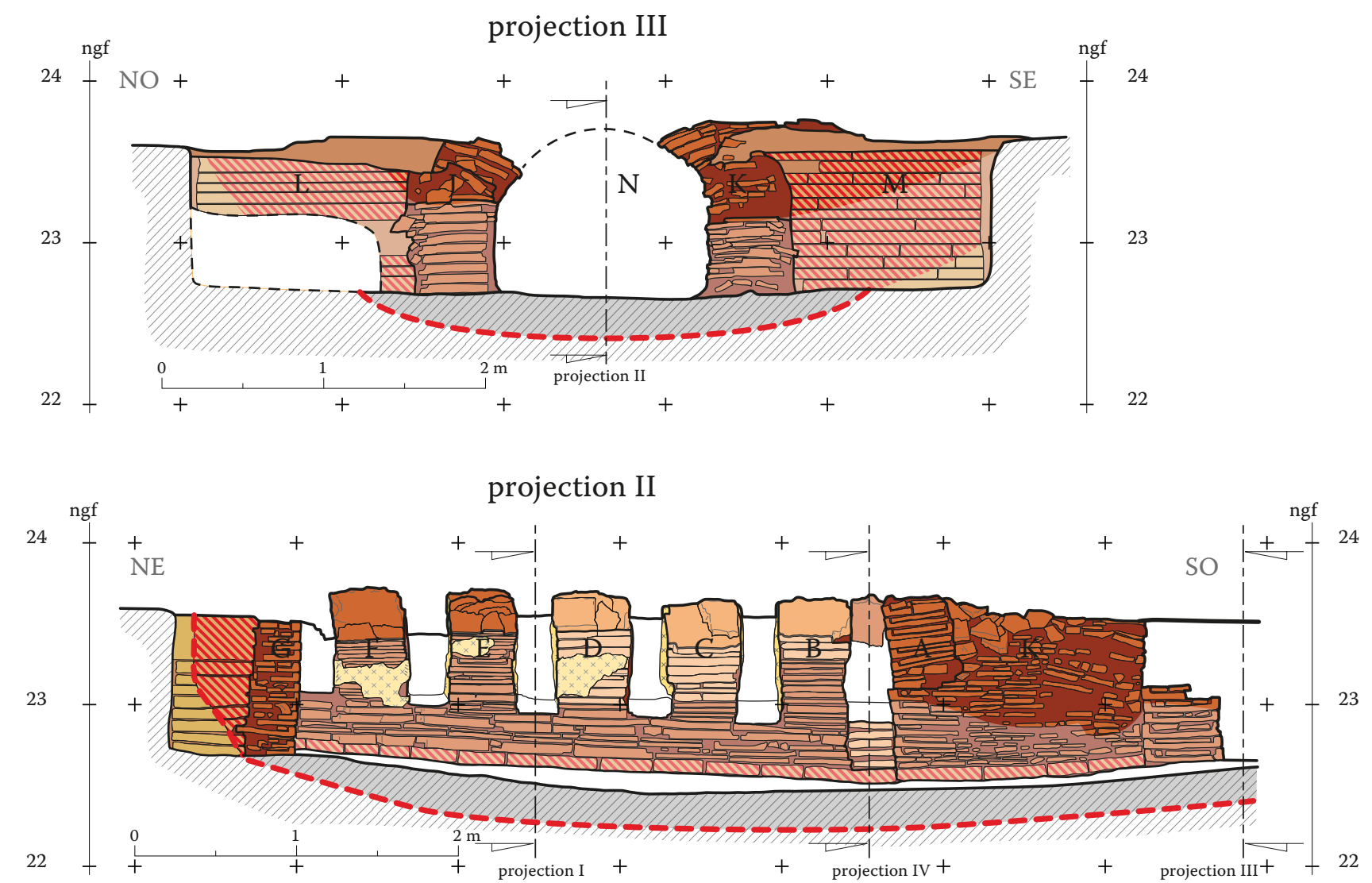

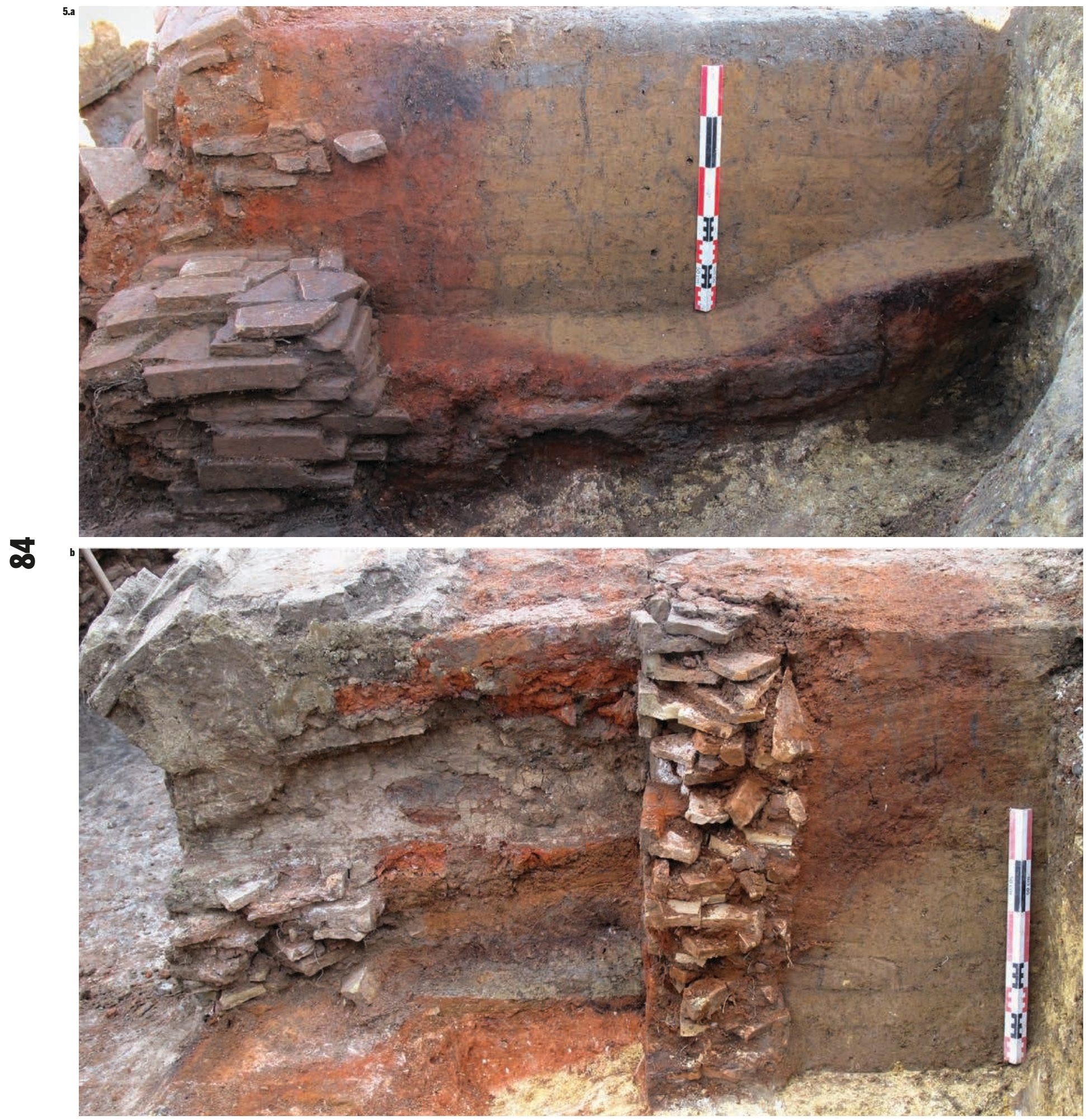

5a.Vue écorchée du

massif d'entrée de

l'alandier du four 2572

(côté est).

b. Coupe des murs

périphériques de la

chambre de chauffe, de la

banquette interne et de

l'élévation de la cloison D

du four 2572 (coupe

SE-NO). 
simultanément avec le corps principal des murs périphériques. Comme le décrit Vitruve (livre II-8) (Vitruve, 1847), l'utilisation de tuiles fragmentées en réemploi dans une construction correspond à une recherche de qualité du matériau. Il préconise ainsi leur usage en maçonnerie car leur utilisation antérieure a permis d'éprouver leur solidité.

\section{Atouts de la terre crue pour un four de tuilier}

Selon sa composition, la brique crue bénéficie d'une résistance à la compression satisfaisante, estimée de 20 à $50 \mathrm{~kg} / \mathrm{cm}^{2}$. Et bien que sa résistance à la traction, à la flexion et au cisaillement soit très faible, la terre crue offre des propriétés mécaniques proches de celles de certains bétons. Comparée à la terre cuite, la terre crue contient beaucoup d'eau. Lors de la chauffe, l'inertie thermique du matériau favorise une diffusion lente de la chaleur dans la masse. Par conséquent, tout au long de la montée en température, la chaleur se propage dans des couches toujours humides produisant des dégazages qui occasionnent des microfissures. Les fissures créent une surface pulvérulente qu'il faut protéger. Les briques crues restent donc plus sensibles aux hautes températures que les terres cuites. Par ailleurs, l'action répétée des cuissons intenses entraîne une induration des briques exposées aux flammes qui acquièrent sur les premiers centimètres des caractéristiques équivalentes aux terres cuites.

La composition de la terre utilisée pour le façonnage des briques à Mours est de nature variable. D'après l'étude de C. Cammas (Cammas, 2015), réalisée sur des prélèvements de briques crues du four 2572, trois types différents sont observés : les briques « jaunes » produites à partir de limons argileux (BT), les briques « gris-verdâtres » montrant un apport en limons carbonatés et enfin les briques « grises » qui sont riches en sables quartzeux et argiles. On peut y voir une volonté du briquetier d'assembler différentes qualités de terre pour optimiser sa production. Les ressources naturelles à proximité expliquent aussi cette diversité. Les versants des buttes stampiennes peuvent, par l'érosion et le colluvionnement, produire des terres riches en sable quartzeux. Les limons de plateau, résultant des dépôts lœessiques bien conservés dans le nord de l'île-de-France, ont subi une pédogénèse qui modifie leur teneur en argile sur l'ensemble de leur stratigraphie. Il n'est donc pas impossible que la quantité de carbonate, de quartz ou d'argile dans la composition des briques soit le fait de sources d'approvisionnement variées ou de la strate exploitée à un moment donné. La fouille n'a pas permis d'identifier les gisements des matières premières. Néanmoins, la variété de certains minéraux peut suggérer l'existence de plusieurs zones d'exploitation simultanées. L'observation nous indique également que ces terres reçoivent un ajout en paille hachée et sont finement malaxées. Le mélange chargé en eau est moulé à l'état pâteux dans un cadre lui donnant des dimensions standardisées de 40 × 29,7 x $6 \mathrm{~cm}$. Ce module rectangulaire est le même que certaines briques cuites utilisées dans la chambre de chauffe du four 2572. Cela nous laisse penser que la fabrication des briques crues ou cuites est simultanée mais que les éléments « crus » sont simplement extraits de la chaîne de production avant leur cuisson.

Dans le four 2572, la terre crue se retrouve dans des parties massives de la construction, notamment dans l'alandier et dans les banquettes de la chambre de chauffe décrites plus haut. Elle se trouve également dans les murs internes du four et dans les murs périphériques [ill. 4a et ill. $5 a$ et b]. Ces élévations sont montées en briques crues posées en panneresse et liées avec un joint mince de moins d'un centimètre de mortier de terre [ill. 6]. La terre crue représente les deux tiers de l'épaisseur de la maçonnerie (l'autre tiers est composé de fragments de tegula). C'est donc le montage des briques crues qui structure toute la construction. D'après les empreintes observées en surface, les murs du laboratoire devaient être construits de la même façon que le soubassement. Si la terre est le matériau idéal pour la base du four, il semble donc qu'il en soit de même pour son élévation. D'autant plus que les murs en élévation ne sont pas destinés à supporter la charge d'exploitation. Ils n'ont pour contraintes que leur poids propre. Leur rôle est de protéger la production lors de sa cuisson en l'isolant de l'environnement extérieur.

La terre n'est pas un très bon isolant à une température normale de $20^{\circ} \mathrm{C}$. Toutefois, à température élevée, la terre est plus isolante que tous les matériaux naturels disponibles à l'époque romaine. Qu'elle soit crue ou cuite, la terre possède une conductivité thermique assez proche, de l'ordre de 0,75 à $1,15 \mathrm{~W} / \mathrm{mK}^{2}$, ce qui est deux fois inférieur aux roches disponibles. De par sa faible conductivité et sa densité, la terre possède une excellente inertie thermique. Ainsi, lors d'une modification de température, la terre atteint le point d'équilibre avec son environnement de façon lente. Elle est donc un atout majeur pour le tuilier qui redoute fortement les changements brusques de températures. Un four doit donc être étanche à l'air et former une masse stable durant la cuisson puis le refroidissement. Le montage de la superstructure en terre crue répond parfaitement à cette exigence de stabilité. Les briques crues montées avec un mortier de terre créent une masse homogène dans tout le volume de la construction. Ces matériaux ont alors une résistance mécanique, une porosité ou des capacités thermiques uniformes. D'autres matériaux pourraient, par leur assemblage, favoriser des dilatations hétérogènes et des ponts thermiques qui modifieraient la structure de manière disparate provoquant sa ruine.

L'exemple du four de tuilier 2572 de Mours montre une construction rigoureuse. La disposition des terres crues ou cuites dans cette architecture répond à un programme exprimée en watt pa mètre-kelvin. C'est la quantité de chaleur qui se propage au travers de $1 \mathrm{~m}^{2}$ de matériau, épais de $1 \mathrm{~m}$, en 1 seconde. 


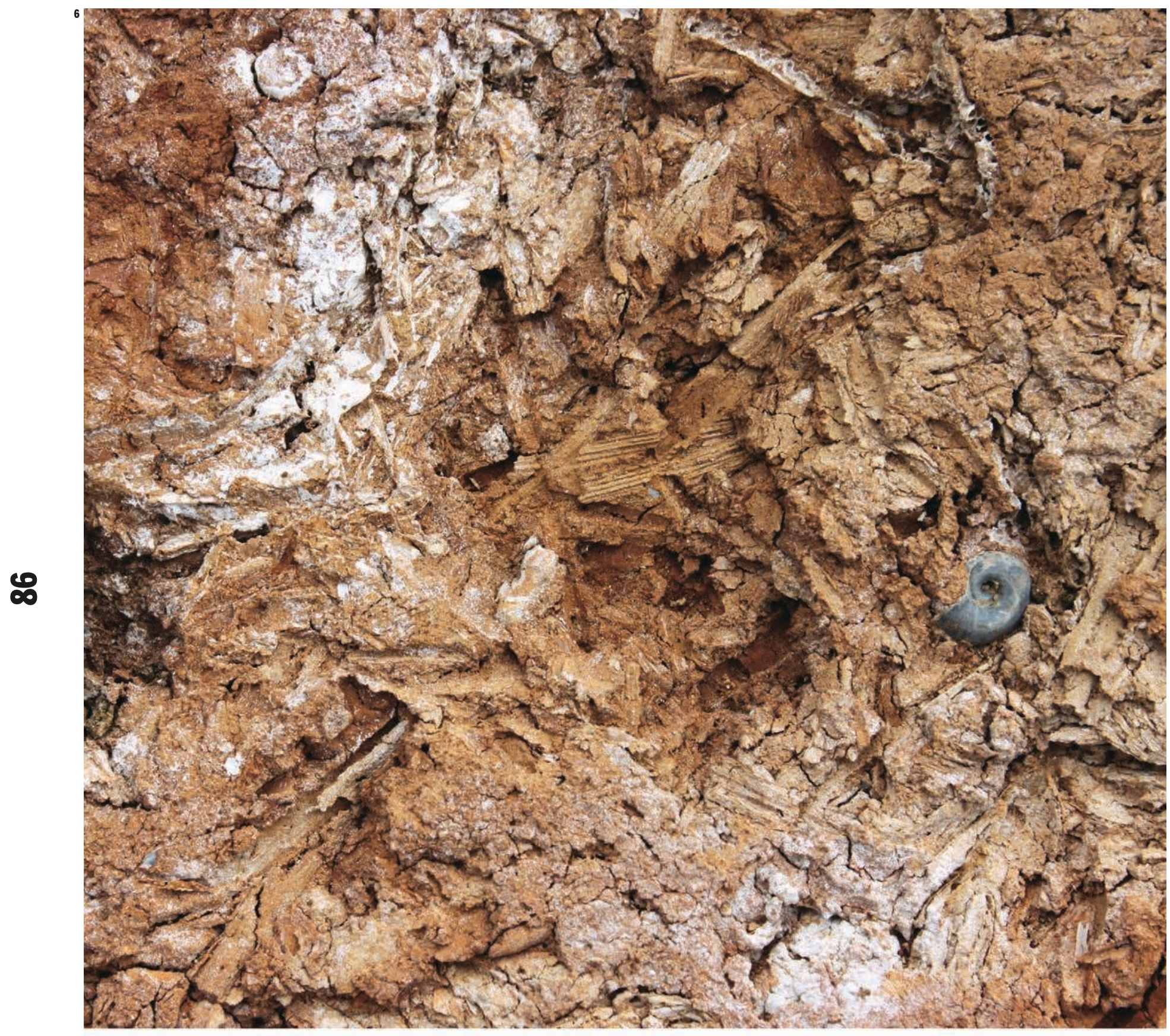

6. Ce mortier de terre,

utilisé pour le montage de

la maçonnerie, situé au

niveau de la sortie de

l'alandier, servait à coller

les tegulæa du conduit de

chauffe du four 2572. 
organisationnel qui tient compte des spécificités techniques de leur fonction. On retrouve ce schéma de principe jusque dans les aménagements comme les banquettes et murs internes, mais également dans les reprises de maçonnerie qui marquent les réparations de la sortie de l'alandier [Fig. 4b-projection IV]. L'assemblage de ces deux matériaux, un qui structure et l'autre qui protège, forme un ensemble porteur qui constitue le principe de la construction du four. Il n'y a pas d'alternance désordonnée des matériaux qui pourrait laisser penser que la terre crue a été choisie par défaut pour combler un manque ponctuel.

La maîtrise architecturale de cet ensemble, sa datation précoce ${ }^{\mathbf{3}}$ et les normes de construction suggèrent un savoir-faire importé ou mis en œuvre selon des directives strictes. Une utilisation ponctuelle ou de courte durée d'un four ne nécessite probablement pas un tel soin dans sa construction. La sélection de ces matériaux en fonction de leur qualité révèle vraisemblablement une volonté de pérenniser cette construction. Cet exemple de structure illustre les connaissances acquises par les constructeurs sur les matériaux à leur disposition. Le matériau terre répond ici à une nécessité et son emploi est bien un choix éclairé.

\section{Références bibliographiques}

Vitruve, 1847, L'architecture de Vitruve, Maufras C. L. (éd.), Paris, C.-L-.F. Panckoucke, 2 vol., 584 p. et 580 p. Disponible sur : http://remacle.org/bloodwolf/ erudits/Vitruve/livre2.htm\#8 (03/og/2015)

AlligRI A., 2015, «Étude archéomagnétique de deux fours de tuilier mis au jour à Mours (95)», in Mondoloni A., Mours, "le Derrière des Moulins », rapport de fouille, Inrap-SRA Île-de-France, vol. 1, p. 151-164.

Alligri A. et RAYMOND P., 2015, «Étude des fours de tuilier 2572 (FR 20) et 1009 (FR 2)», in MoNDOLONI A., Mours, «le Derrière des Moulins », rapport d'opération, Inrap-SRA Île-de-France, vol. 1, p. 127-168.

CAMmas C., 2015, « Expertise micromorphologique sur les briques en terre crue du four de tuilier romain 2572 (FR 20) », in Mondoloni A., Mours, «le Derrière des Moulins », rapport d'opération, InrapSRA Île-de-France, vol. 1, p. 169-172.

CHARLIER F., 2011, Technologie des tuiliers gallo-romains (Gaules et Germanies), Analyse comparative et régressive des structures de production des matériaux de construction en terre cuite de l'époque contemporaine à l'Antiquité, Thèse de doctorat, Histoire Ancienne, Université de Franche-Comté, $856 \mathrm{p}$

HoMAND-ÉTIENNE F., 1986, Comportement mécanique des roches en fonction de la température, Nancy, Éd. de la Fondation scientifique de la géologie et de ses applications (coll. Sciences de la terre. Mémoires, 46), 216-VIII p.-[6] p. de pl.

Le GofF M., Gallet Y, Genevey A., Warmé N., 2002 "On archeomagnetic secular variation curves and archeomagnetic dating ", Physics of the earth and planetary interiors, 134, p. 203-211.

LE Ny F., 1988, Les fours de tuiliers gallo-romains : méthodologie, étude technologique, typologique et statistique, chronologie, Paris, Éd. de la Maison des sciences de l'homme (coll. Documents d'archéologie française, 12$), 142 \mathrm{p}$

MAUNÉ S., SilvérÉANo S., avec la coll. de NewMan C., 2011, « Les productions augusto-tibériennes de l'atelier de potiers de Bastide-Neuve à Vélaux (Bouches-du-Rhône) », in Actes du Congrès international de la Société française d'étude de la céramique antique en Gaule (SFECAG), Arles, 2-5 juin 2011, Marseille, SFECAG, p. 667-69o.

MoNDOLONI A., 2015, Mours, «le Derrière des Moulins », Une structure de crémation au néolithique récent et les occupations protohistorique, antique et médiévale, rapport d'opération, Inrap-SRA Île-de-France, 2015 2 vol., $1148 \mathrm{p}$.

Nguyen T. D., 2009, Étude du comportement au feu des maçonneries de briques en terre-cuite, Approche expérimentale et modélisation du risque d'écaillage, Thèse de Doctorat, Génie civil, Université Paris-Est, Laboratoire de Modélisation et Simulation MultiÉchelle, 154 p. Disponible sur : https://tel.archivesouvertes.fr/tel-oo539872/document (o3/o9/2015)

SCHUlz R. , Fischer B., 2002, Die Ziegeleimanufaktur von Neupotz, Kreis Germersheim, Archäologie in Rheinland-Pfalz, p. 96-98.

TRIMPERT H. A, 2003, Die römischen Ziegeleien in Rheinzabern "Fildelisstraße». Mit studien zum römischen Ziegeleiwesen in den germanischen Provinzen und Raetien, Speyer, Landesamt für Denkmalflege Archäologische Denkmalpflege Amt Speyer, $300 \mathrm{p}$. 